06

\title{
Characterization of the Optical Nonlinearities of Silver and Gold
} Nanoparticles

\author{
(C) R.A. Ganeev
}

The Guo China-US Photonics Laboratory, Changchun Institute of Optics, Fine Mechanics and Physics, Chinese Academy of Sciences, 130033 Changchun, China

e-mail: rashid_ganeev@mail.ru

Received March 28, 2019

Revised March 28, 2019

Accepted April 23, 2019

The synthesis of nanostructured materials took much attention due to their advanced optical and nonlinear optical properties, which can be used in various areas of communications, optics, laser physics and medicine. During last two decades, special attention was given to the nonlinear optical properties of the nanoparticles (NPs) of variable morphology. Here we review the recent studies of the nonlinear optical properties of silver and gold NPs. We discuss the Z-scans and pump-probe transient absorption studies allowing determining the nonlinear refractive indices, nonlinear absorption coefficients and electron-phonon interaction times. The analysis of the low- and highorder harmonic generation in the laser-produced plasmas containing Ag NPs and Au NPs is also presented.

Key words: nonlinear optics, silver and gold nanoparticles.

DOI: $10.21883 /$ OS.2019.09.48201.123-19

* Полная версия статьи опубликована в английской версии журнала. 